\title{
Viscoelastic properties of tablets from Osborne fractions, pentosans, flour and bread evaluated by creep tests
}

\author{
Anayansi Escalante-Aburto ${ }^{1}$, Juan de Dios Figueroa-Cárdenas ${ }^{2}$, José Juan Véles-Medina ${ }^{2}$, \\ Néstor Ponce-García ${ }^{3,4}$,Zorba Josué Hernández-Estrada ${ }^{5}$, Patricia Rayas-Duarte ${ }^{5}$, and Senay Simsek ${ }^{6}$ \\ ${ }^{1}$ Department of Nutrition, University of Monterrey, Ignacio Morones Prieto Avenue 4500 west, San Pedro Garza García, \\ Nuevo León, 66238, México \\ ${ }^{2}$ Center of Research and Advanced Studies - Querétaro (CINVESTAV-IPN), Libramiento Norponiente 2000, \\ Fracc. Real de Juriquilla, Querétaro, Querétaro, 76230, México \\ ${ }^{3}$ Center of Biotechnology FEMSA, School of Engineering and Sciences, Tecnológico de Monterrey, Eugenio Garza Sada Avenue \\ 2501 South, 64849, Monterrey, Nuevo León, México \\ ${ }^{4}$ UAEMex Campus Universitario 'El Cerrillo', Faculty of Agricultural Sciences, Piedras Blancas s/n, Toluca, Estado de México, \\ 50200, México \\ ${ }^{5}$ Robert M. Kerr Food and Agricultural Products Center, Oklahoma State University, 123 FAPC, Stillwater, OK 74078-6055, USA \\ ${ }^{6}$ Department of Biochemistry and Molecular Biology, North Dakota State University, Harris Hall 224, Dept 7670, PO Box 6050, \\ Fargo, ND 58108-6050, USA
}

Received October 8, 2016; accepted June 19, 2017

\begin{abstract}
$\mathrm{A} \mathrm{b} \mathrm{s} \mathrm{t} \mathrm{r} \mathrm{a} \mathrm{c} \mathrm{t.} \mathrm{Little} \mathrm{attention} \mathrm{has} \mathrm{been} \mathrm{given} \mathrm{to} \mathrm{the} \mathrm{influence}$ of non-gluten components on the viscoelastic properties of wheat flour dough, bread making process and their products. The aim of this study was to evaluate by creep tests the viscoelastic properties of tablets manufactured from Osborne solubility fractions (globulins, gliadins, glutenins, albumins and residue), pentosans, flour and bread. Hard and soft wheat cultivars were used to prepare the reconstituted tablets. Sintered tablets (except flour and bread) showed similar values to those obtained from the sum of the regression coefficients of the fractions. Gliadins and albumins accounted for about $54 \%$ of the total elasticity. Gliadins contributed with almost half of the total viscosity (45.7\%), and showed the highest value for the viscosity coefficient of the viscous element. When the effect of dilution was evaluated, the residue showed the highest instantaneous elastic modulus (788.2 MPa). Retardation times of the first element $\left(\lambda_{1} \sim 3.5 \mathrm{~s}\right)$ were about 10 times lower than the second element $\left(\lambda_{2} \sim 39.3 \mathrm{~s}\right)$. The analysis of compliance of data corrected by protein content in flour showed that the residue fraction presented the highest values. An important contribution of non-gluten components (starch, albumins and globulins) on the viscoelastic performance of sintered tablets from Osborne fractions, flour and bread was found.

K e y w o r d s: viscoelasticity, creep test, Osborne fractions, fluor, bread
\end{abstract}

*Corresponding author e-mail: anayansi.escalante@udem.edu

\section{INTRODUCTION}

Different investigations have reported that the unique viscoelastic properties of wheat grains reside primarily in the gluten forming storage proteins of its endosperm (Gianibelli et al., 2001; Greenwood and Ewart, 1975; Lindsay and Skerritt, 1999). Many parameters such as the quality of flour protein, the amount of gluten, proportion of gluten proteins, and the type of subunits present in the flour are evaluated to predict the quality of dough and bakery products (Saxena et al., 1997). However, little attention has been given to the evaluation and influence of non-gluten components, such as pentosans and starch, on the viscoelastic properties of dough, the bread making process and their products.

D'Appolonia and Gilles (1971) mentioned that pentosans are closely associated with proteins, especially with the gluten fraction of wheat flour, hence these components could be related with the viscoelastic properties during the bread making process. For indirect measurement of wheat properties associated with gluten and non-gluten components, Hernández-Estrada et al. (2012) proposed the creep

(C) 2017 Institute of Agrophysics, Polish Academy of Sciences 
test and the use of the generalised Kelvin-Voight model methodology that allowed the separation of rheological parameters like elastic, viscoelastic and viscous flow characteristics. The plastic work of wheat kernels may be related to water, lipids, pentosans and other carbohydrates that are important for dough extensibility (Figueroa et al., 2009). Non-gluten components of dough, like albumins, globulins, starch and pentosans, showed lower elastic moduli than dough (Hernández-Estrada et al., 2014, 2017). In general, viscoelastic behaviour of wheat kernels and their doughs are most likely explained by the interactions of all of their gluten and non-gluten components that form unique systems (Ponce-García et al., 2016). Thus, the study of viscoelastic properties of other wheat components besides gluten alone justifies their evaluation using different methods that would complement the body of knowledge in this field.

Figueroa et al. (2016) indicated that starch granules, water soluble pentosans and non-gluten components may not be considered as inert filler, since it has been clearly shown that those components are also responsible for the viscoelastic behaviour of sintered tablets made from wheat products such as flour, dough and bread.

The aim of this study was to evaluate the viscoelastic properties of sintered tablets of proteins obtained from Osborne solubility fractions (globulins, gliadins, glutenins, albumins and residue), pentosans, flour and bread from two wheat classes, evaluated by creep tests.

\section{MATERIALS AND METHODS}

Samples of four cultivars of hard red spring wheat (HRS): Len, Butte, Era and Coteau, and of two cultivars of soft red wheat (SRW): Adder and Fairfield, were tested in this study.

The grain samples were tempered at room temperature on the lab bench in plastic bottles. After measuring the moisture of the wheat with NIR using a FOSS Infratec 1241 grain analyser (FOSS, Eden Prairie, MN), additional water was added to bring the moisture to $12 \%$. The samples were shaken every hour for $3 \mathrm{~h}$ and then rested at room temperature for approximately $16 \mathrm{~h}$ prior to milling. Tempered wheat samples were milled using a Brabender Quadrumat Jr. (C.W. Brabender, South Hackensack, NJ) laboratory mill. Straight grade flour was blended and screened through an $84 \mathrm{SS}$ sieve to remove foreign material. The bread was prepared using a $2 \mathrm{~h}$ fermentation schedule to avoid overfermentation (AACCI, 2011). Bread loaves were sliced to $10 \mathrm{~mm}$ thick and placed in a single layer on wire drying racks. The bread was dried in two stages -1 ) overnight on the laboratory bench at approximately $25^{\circ} \mathrm{C}$ and $35-40 \%$ relative humidity, followed by grinding in a food chopper, and 2) freeze dried (Labconco Freezone 4.5, serial 061162977S, Kansas, Missouri) during $72 \mathrm{~h}$ at $-53^{\circ} \mathrm{C}$ and $0.050 \mathrm{mBar}$, to reduce the moisture content to about $1 \%$.
Solvent solubility extraction was conducted according to the modified Osborne fractionation procedure of Chen and Bushuk (1970) in the wheat flour. Samples of flour with $\mathrm{NaCl}(0.5 \mathrm{M})$ were stirred and centrifuged, the supernatant (albumins and globulins) was dialysed with water to precipitate the globulin fraction. The pellet left in the tube (sediment) was mixed with $70 \%$ aqueous ethanol, stirred and centrifuged; the gliadin fraction was obtained in the supernatant by dialysis to remove ethanol. The pellet left on the tube was stirred with acetic acid $(0.05 \mathrm{M})$ and centrifuged, the supernatant contained the acid soluble glutenin fraction. The pellet contained the residue, mostly starch containing acid insoluble glutenin. All the Osborne protein fractionation was carried out at $4^{\circ} \mathrm{C}$, and all the centrifugations were at $5000 \times \mathrm{g}$ for $30 \mathrm{~min}$.

Fractions were freeze dried (Labconco Freezone 4.5, serial $061162977 \mathrm{~S}$, Kansas, Missouri) during $72 \mathrm{~h}$, at $-53^{\circ} \mathrm{C}$ and $0.050 \mathrm{mBar}$, and stored in tightly closed containers at $5^{\circ} \mathrm{C}$ until needed for analysis (approximately two weeks). Water soluble pentosans (WSP) were calculated by difference using the recovery of the fractions (Figueroa et al., 2016).

Tablets of $4.2 \mathrm{~mm}$ height and $8.07 \mathrm{~mm}$ diameter (contact area of $51.52 \mathrm{~mm}^{2}$ ) were sintered from Osborne protein fractions, flour and bread. For each tablet, $300 \mathrm{mg}$ of sample was weighed into a $10 \mathrm{ml}$ beaker sealed with parafilm and placed in a controlled temperature chamber at $20^{\circ} \mathrm{C}$ for $2 \mathrm{~h}$. The tablet-forming die of hardened steel had an inside diameter of $7.95 \mathrm{~mm}$, and a length of $31.69 \mathrm{~mm}$. Two hardened steel dowels were used to apply the pressure necessary to form the tablets. The lower dowel (5.54 $\mathrm{mm}$ long) was inserted into a loose-fitting cylinder, 31.69 $\mathrm{mm}$ high, and the die was placed on top of this cylinder with the dowel of $43.23 \mathrm{~mm}$ long positioned into the die. The sample was transferred into the die, the upper dowel placed into the lower die and the set positioned onto a table with a hydraulic press (Trupper 501, $50 \mathrm{t}$ capacity). The load on the die was gradually increased to reach $25 \mathrm{t}$ and maintained for $5 \mathrm{~min}$ before removing the tablet from the die. Reconstituted tablets were also prepared, containing the same percentage $(w / w)$ of the fractions obtained. Six tablets were obtained for each fraction.

In creep experiments, the material is subjected to dead loads under constant strain and the deformation as a function of time is observed (Mohsenin, 1986). Once the tablets were obtained and before the test began, the height and diameter were measured using a caliper (Model CD-6 CS, Mitutoyo Corp., Japan) in order to calculate the applied strain and the contact area of each tablet.

The method used to measure viscoelastic properties by creep test was according to Hernández-Estrada et al. (2012) with some modifications. Briefly, before the test, 4 tablets with about $4 \%$ moisture content were placed in a controlled temperature chamber at $25^{\circ} \mathrm{C}$ for $2 \mathrm{~h}$ to reduce variance in the results. A Texture Analyzer (Texture Technologies 
Corp. TA-XT2) with a $10 \mathrm{~mm}$ diameter stainless steel probe (TA-510) was used to measure the tablets deformation. The tablets were loaded in compression until reaching $70 \mathrm{~N}$ with a constant loading rate of $0.1 \mathrm{~mm} \mathrm{~s}^{-1}$. Load $(\mathrm{N})$ and displacement $(\mathrm{mm})$ data were acquired at 10 points $/ \mathrm{s}$, the stress was held constant for $300 \mathrm{~s}$, and the displacement data was used to calculate strain dividing the deformation by the initial height of the tablets. The test was performed once on each tablet to obtain four curves and data were extracted for calculations.

Mechanical models consisting of springs and dashpots are used to explain and interpret the rheological behaviour of linear viscoelastic materials. The spring obeys the Hooke law and the dashpot the Newton law. In the Kelvin-Voigt model, the elements are forced to move together at constant rate (Mohsenin, 1986), a three elements model that follows the Eq. (1):

$$
\varepsilon(t)=\frac{\sigma_{0}}{E_{0}}+\frac{\sigma_{0}}{E_{1}}\left(1-e^{-t / \lambda_{1}}\right),
$$

where: $\varepsilon(t)$ is the strain (\%) as a function of time $t(\mathrm{~s}), \sigma_{0}$ is the applied stress, $E_{0}(\mathrm{MPa})$ is the elastic modulus of the Hookean body; the retardation time $\left(\lambda_{1}=\mu_{1} / \mathrm{E}_{1}\right)$ that in the case of creep is $(1-1 / e)$ or about $63 \%$ of the time for total strain; $E_{1}$ is the elastic modulus and $\mu_{1}\left(\mathrm{MPa} \mathrm{s}^{-1}\right)$ is the viscous coefficient of the dashpot in the Kelvin-Voigt element.

A four-element Burger model resulted of a combination of the Maxwell and Kelvin-Voigt models and is expressed in Eq. (2):

$$
\varepsilon(t)=\frac{\sigma_{0}}{E_{0}}+\frac{\sigma_{0}}{E_{1}}\left(1-e^{-t / \lambda_{1}}\right)+\frac{\sigma_{0}}{\mu_{0}} t .
$$

The first term of Eq. (2) represents the instantaneous elastic deformation of the sample, the second term is related to the combined effect of viscoelasticity, and the third term expresses the viscous effect and the viscosity coefficient $\left(\mu_{0}, \mathrm{MPa} \mathrm{s}^{-1}\right)$.
According to Mohsenin (1986), to obtain a 6-element model, another Kelvin-Voigt unit must be added to the Burger model (Fig. 1).

To obtain the results in terms of uniaxial creep compliance, the equation suggested by Hernández-Estrada et al. (2012) was applied (Eq. (3):

$$
D(t)=\frac{\varepsilon(t)}{\sigma_{0}}=D_{0}+D_{1}\left(1-e^{-t / \lambda_{1}}\right)+D_{2}\left(1-e^{-t / \lambda_{2}}\right)+\frac{t}{\mu_{0}},
$$

where: $\varepsilon$ - strain (\%), $\mathrm{t}$ - time (s), $\sigma-$ stress (MPa), $\lambda$ - retardation time (s), and $D$ - compliance $(1 / \mathrm{Pa}), D_{0}-$ instantaneous compliance or compliance at zero time (1/ $\mathrm{Pa}), D_{1}, D_{2}$ - compliance of 1 st and 2 nd retarded elastic element $(1 / \mathrm{Pa})$.

Analysis of variance (ANOVA) was performed using the General Linear Model procedure of SAS Statistical Software, version 9.3. Multiple comparison of the means was performed using the Duncan Multiple Range test at $\alpha=0.05$ level. Nonlinear regression analyses were performed with OriginPro 9 (OriginLab Corporation, Northampton, MA, USA).

\section{RESULTS AND DISCUSSION}

The evaluated protein fractions reached a quasi-steady state at $100 \mathrm{~s}$ after the application of constant stress. Usually, creep test performed on intact wheat kernels required more time (up to 200-1200 s) to reach the quasi equilibrium. The coefficients obtained by the creep curves fitted with the generalised Kelvin-Voigt model with six elements showed that gliadins and albumins represent about $54 \%$ of the total elasticity, however, gliadins showed the highest elastic modulus $\left(E_{0}\right)$ among all the evaluated fractions (Table 1). Glutenins contributed with $17 \%$ of the total elasticity $(E \%)$ showing an $E_{0}$ of $1391.2 \mathrm{MPa}$, significantly higher than those reported by Hernández-Estrada et al. (2012). Those authors found an average value of $242 \mathrm{MPa}$ for the elastic moduli of HMW-GS and LMW-GS from thirty-four wheat lines,

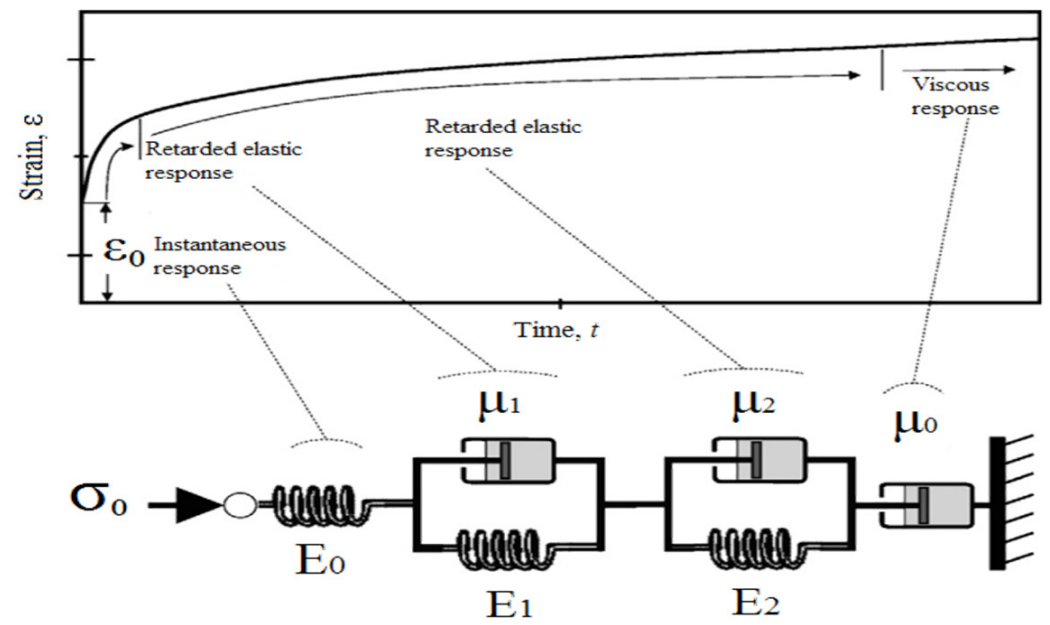

Fig. 1. Analogous mechanical representation of the generalised Kelvin-Voigt model with 6 elements (Hernández-Estrada et al., 2012). 


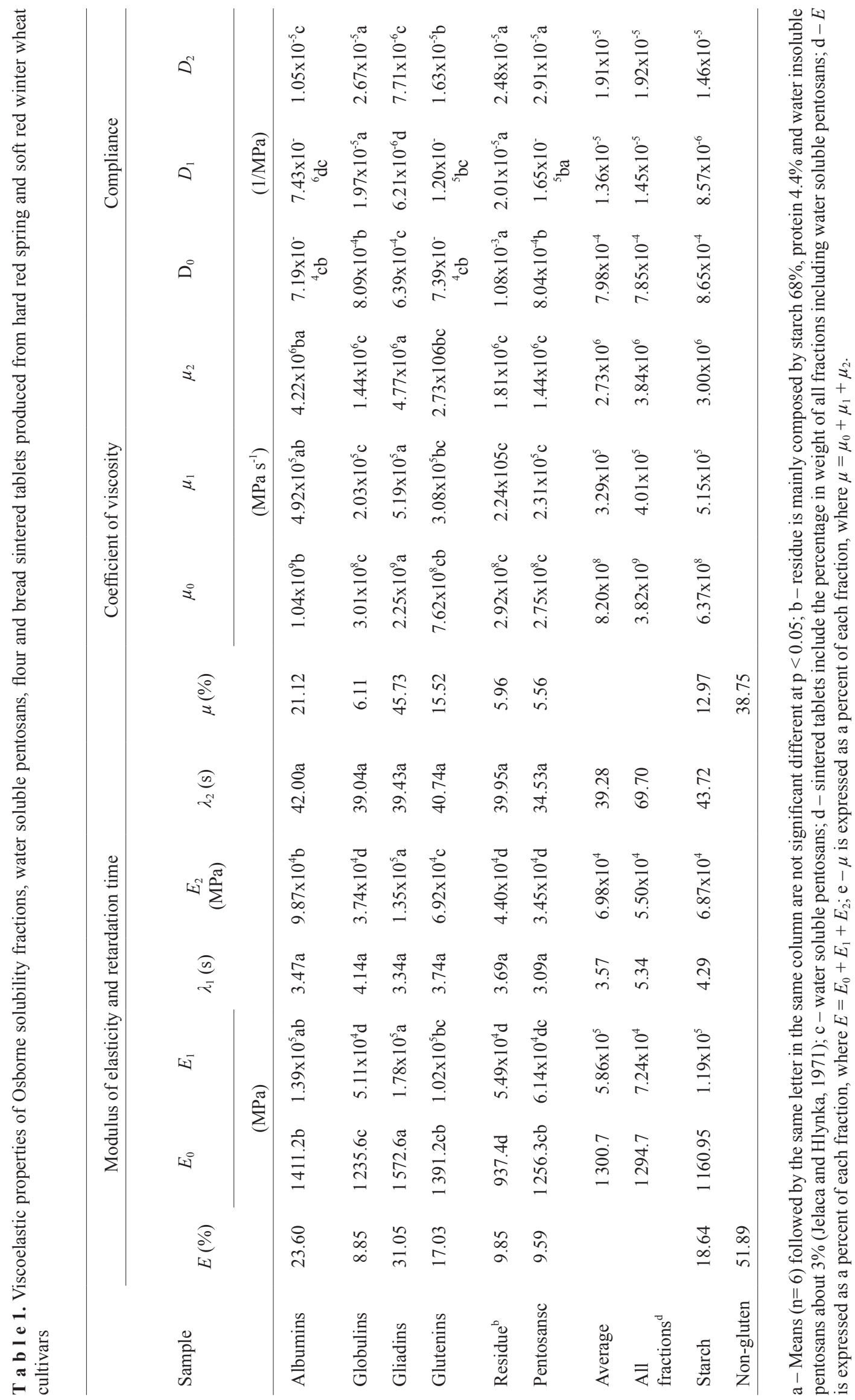


this value being lower than that found in this study. Perhaps there was an additive effect of the modulus from the different glutenin subunits that was expressed in the complete fraction. However, when starch was evaluated separately, higher values were showed for the instantaneous elastic moduli, similar to those obtained by albumins and gliadins. Staniforth and Patel (1989) evaluated the creep compliance behaviour of direct compression excipients from different materials, suggesting that starch undergoes a high degree of elastic deformation at different stress levels.

The retarded modulus of elasticity of the second element $\left(E_{2}\right)$ showed lower values in almost all fractions when compared with $\mathrm{E}_{1}$ (Table 1). Gliadins showed the highest values for both moduli $\left(E_{1}\right.$ and $\left.E_{2}\right)$. Retardation times $\lambda_{1}(\sim 3.5 \mathrm{~s})$ were lower than $\lambda_{2}(\sim 39.3 \mathrm{~s})$, but no significant differences were detected among the fractions. Shorter retardation times were detected in all Osborne fractions and water soluble pentosans, when compared with those reported by Figueroa et al. (2011) and Hernandez-Estrada et al. (2012) on intact wheat kernels and glutenin subunits, respectively. The latter means that differences between materials can be measured using this methodology, applying rheological models that detect differences among samples. Thus, wheat proteins behave in a different way depending of their molecular structure, which could be related with the quality parameters of their products.

Dobraszczyk and Morgenstern (2003), using the results obtained by Bloksma and Bushuk (1988), described the experimental measurements for stress relaxation tests in different doughs. The curves did not show the exponential decay typical for a single relaxation time, indicating a wide range of relaxation times. Their results indicated that molecular mechanisms are involved for the relaxation process and deformation processes within dough, which can be related to the wide molecular weight distribution of gluten.

In this study, gliadins contributed with almost half of the total viscosity (45.7\%), and showed the highest value for the viscosity coefficient of the viscous element $\left(\mu_{0}\right.$, dashpot). With respect to the first and second retarded elastic deformation elements $\left(\mu_{1}\right.$ and $\left.\mu_{2}\right)$, albumins and gliadins showed the highest values (Table 1 ). The viscosity coefficients displayed wide variation, there were reductions by factors of 1000 for $\mu_{1}$, and 100 for $\mu_{2}$. Gliadins showed the highest values for $\mu_{0}, \mu_{1}$, and $\mu_{2}$, which describes the plasticiser effect of gliadins and its function as a viscous material promoting the extensibility of gluten (Belton, 1999). Plastic components seem to be more related to non-gluten factors (Maucher et al., 2009). Ponce-García et al. (2016) noted that some low molecular weight glutenin subunits (LMWGS), gliadins, lipids, pentosans and other carbohydrates act as plasticisers for dough extensibility. It was remarkable that the albumin fraction also showed higher viscosity coefficients $\left(\mu_{1}, \mu_{2}\right)$, significantly contributing to the vis- cosity of the system. Glutenins showed $1 / 3$ of the viscosity when compared with gliadins, in fact, the glutenin fraction showed similar values for $E \%$ and $\mu \%$.

The residue showed the highest instantaneous compliance $\left(D_{0}\right)$. When $D_{1}$ and $D_{2}$ (compliance of the first and second retarded elastic elements) were evaluated, globulins, residue and water soluble pentosans presented the highest values (Table 1). According to Hernández et al. (2012), compliances obtained from creep test in kernels are correlated to some quality parameters such as SDS, SDS index, mixing time, alveograph deformation energy of dough, tenacity-extensibility ratio and tenacity.

The estimated parameters of the Kelvin-Voigt model for creep test coefficients from the Osborne solubility fractions and water soluble pentosans were corrected by weight (Table 2). The correction was made in order to compare the parameters and to observe the dilution factor effect that could exist among the fractions. When the extraction was performed, the residue showed the highest weight, as expected $(86.1 \%)$, of all the fractions, followed by albumins and gliadins. It can be seen that the sum of gliadins and glutenins accounted for only $6.9 \%$ from the total flour weight.

In sintered tablets, gliadins, albumins and glutenins showed the highest instantaneous elastic moduli (Table 1). Nevertheless, when the effect of dilution was evaluated (Table 2), the residue showed the highest instantaneous elastic modulus $E_{0}(788.2 \mathrm{MPa})$, with similar values in Tables 1 and 2, perhaps by the largest proportion obtained on the Osborne fractionation. In addition, the residue fraction presented the highest values of elastic moduli $\left(E_{1}\right.$ and $E_{2}$ ) when compared with the other fractions. In creep-compliance curves, starch shows a region representing a higher elastic deformation during compression, represented by spring stretching in the Maxwell unit (Stainforth and Patel, 1989). The dough strength has been attributed to glutenin fractions (Khatkar and Schofield, 1997), however, in their study the water effect on each fraction was not evaluated. It may be seen that the low contribution of glutenins to the elasticity and the low values of the elastic components were a consequence of their characteristics, such as glutenins subunit size, polarity and number of cysteine residues, influencing the capability of disulphide bonds formation, which are necessary to conform the glutenin polymer structure (Žilić et al., 2011). The estimated retardation times $\left(\lambda_{1}\right.$ and $\lambda_{2}$ ) were not statistically different among the fractions.

When viscosity coefficients were analysed, the residue fraction showed the highest values for $\mu_{0}, \mu_{1}$ and $\mu_{2}$, contributing with more than a half of the total viscosity (58.5\%), followed by gliadins $(26.5 \%)$ and albumins $(9.3 \%)$ fractions (Table 2). The coefficient of viscosity $\mu_{0}$, representing the viscous response of the sample, was also the highest in the residue fraction. 


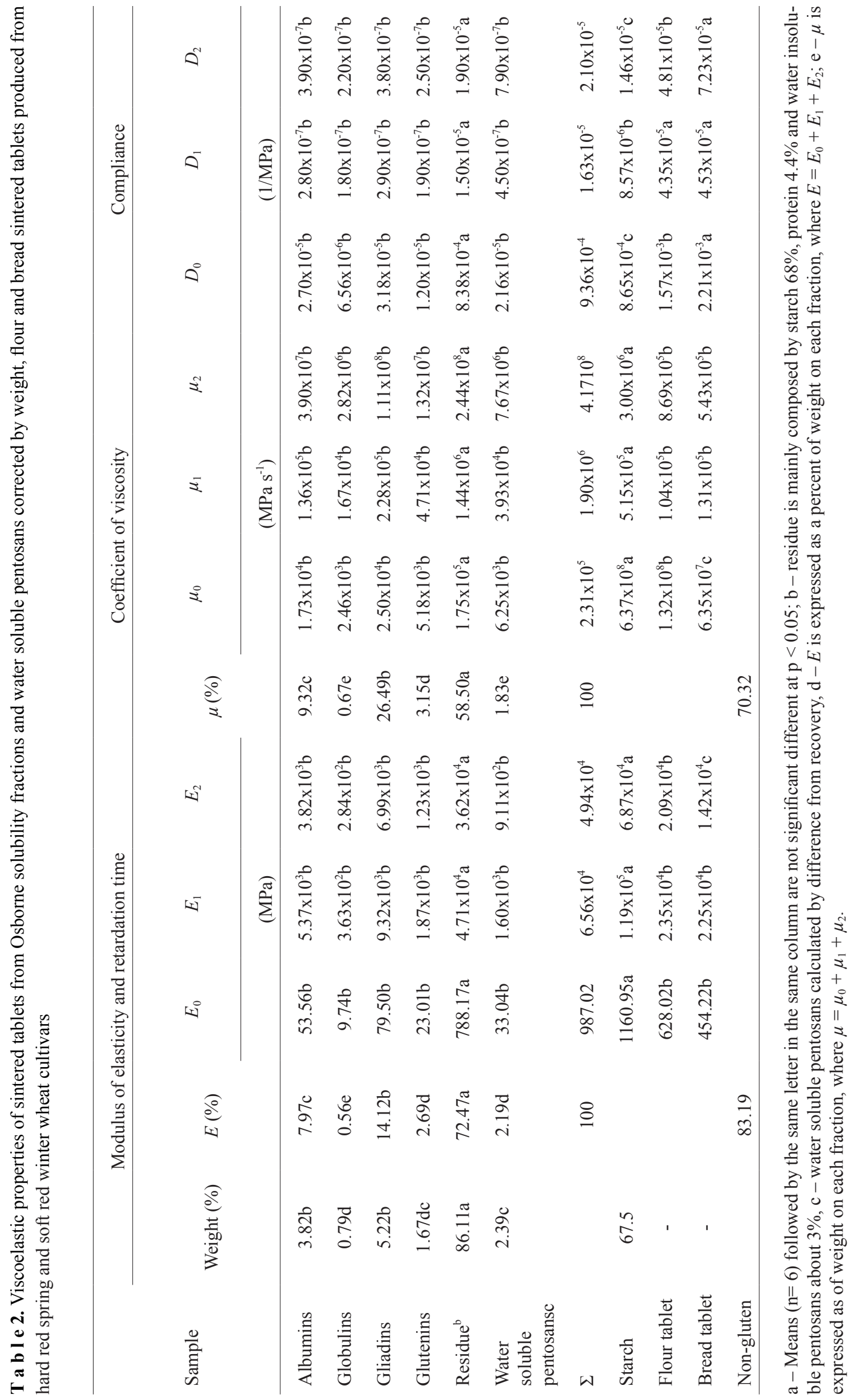


When compliances were analysed, the same trend was present, where the residue fraction showed the highest values among the other protein fractions, with magnitude of $1.0 \times 10^{-2} \mathrm{MPa}$ (Table 2). Haddad et al. (2001) demonstrated that endosperm of different wheat classes showed mechanical properties that could be explained by rheological models. They tested wheat endosperm in parallelepiped shaped form and described a viscoelastic behaviour with a threshold similar to that observed in other materials such as metals and polymers.

The latter proved that the all the wheat components interact among one another to give its unique viscoelastic characteristics. Since the residue was composed mainly of starch, in our study we isolated the rheological behaviour from the almost pure fraction, showing the important contribution of this and other non-gluten components to dough performance and to the quality characteristics of the products.

The estimated parameters of the Kelvin-Voigt model for creep test coefficients, from the Osborne solubility fractions and water soluble pentosans, were corrected by protein content in flour (Table 3). To our knowledge, there were no previous data to compare the viscoelastic parameters obtained in this study with. Nevertheless, it can be seen that the highest protein content is found in the gliadins fraction, followed by glutenins and albumins fraction, respectively. When the data were examined after the correction by protein in flour, it was observed that the highest elasticity value was provided by the gliadins fraction.

The instantaneous response $\left(E_{0}\right)$ and the retarded elastic response elements $\left(E_{1}\right.$ and $\left.E_{2}\right)$ of gliadins were the highest $(57.4 \%)$, and were statistically different when compared with the other fractions (Table 3). The same trend was observed for the viscosity coefficients, since that fraction contributed with $72.2 \%$ of the total viscosity, almost 10 times the viscosity of the glutenins fraction. Li et al. (2006) studied the polymer conformation of structure of wheat proteins and noted that the presence of $\beta$-sheet structure and content in gliadins and glutenins are quite different, the glutenins being those of lower content. Those authors proposed that gliadin and soluble glutenin molecules mainly form $\beta$-turn structures, and insoluble glutenins in gel proteins form a $\beta$-sheet structure, which seem to be an important contributor to the formation of a network structure and, subsequently, affected the elastic and long-time relaxation properties of gluten proteins.

It was evident, when Table 1 and Table 3 were compared, that protein content in each fraction influences significantly the viscoelastic parameters, since the ratio of glutenins and gliadins is 1:4. These results were according to our previous study (Figueroa et al., 2016), where gliadins fraction showed the highest elastic and viscosity coefficients obtained by relaxation tests. Glutenins fraction represented only 8.7 and $6.5 \%$ of elasticity $(E \%)$ and viscosity $(\mu \%)$, respectively.
The residue and albumin fractions provided similar values of elasticity $(E \%)$, however, the residue fraction presented higher instantaneous elastic response of the $E_{0}$ element. Statistical differences were shown among the fractions, which evidenced the specific rheological characteristics of each group of molecules (Table 3 ).

The sum of the elastic moduli for flour $\left(E_{0}+E_{1}+E_{2}\right)$ was $4.50 \times 10^{4} \mathrm{MPa}$, however, the sums of the elastic moduli of the total proteins fraction (fractions) and starch in flour amounted to $5.49 \times 10^{4}$ and $1.88 \times 10^{5} \mathrm{MPa}$, respectively. Thus, starch showed a higher elastic modulus $\left(E_{0}\right)$ than flour and bread, respectively. Our previous study noted that total proteins contributed with only $2.03 \mathrm{MPa}$ and that the higher contribution to the elastic performance was provided by starch, which agrees with the results presented in this study. The contribution of water soluble pentosans was relatively low, as expected, since their protein content is minimum; those compounds showed values of $E \%=$ 0.93 and $\mu \%=0.49$ from the total elasticity and viscosity, respectively (Table 3). However, even when those components show lower viscosity performance, their functionality and interaction with gluten proteins increase, as demonstrated by Santos et al. (2005). They noted that during gluten formation, the water soluble pentosans (WSP) caused a reinforcement of the network, forming structural rearrangements with other components of flour, which have effects on gluten functionality (probably by the differences on the polysaccharide size), increasing the elastic moduli and viscosity coefficients.

When compliances of Osborne extraction were analysed, the residue fraction showed the highest values for $D_{0}, D_{1}$ and $D_{2}$, followed by gliadins and glutenins fractions. However, flour and bread presented the highest compliances when compared with the fractions and WSP.

\section{CONCLUSIONS}

1. An important contribution of non-gluten components, such as starch, albumins and globulins to the viscoelastic performance of sintered tablets from Osborne fractions, flour and bread was found.

2. Reconstituted tablets (albumins, globulins, gliadins, glutenins, residue and water soluble pentosans) showed similar values to those obtained from the sum of the regression coefficients of the fractions.

3. Starch fraction showed remarkable viscoelastic behaviour, and as a major component of flour contributed greatly to its functionality.

4. Gliadins, albumins and glutenins showed the highest contribution to the elasticity, viscosity and compliance of sintered tablets.

5. When compliance data corrected by protein content in flour were analysed, the residue fraction showed the highest values, demonstrating that in this fraction strain increases for a constant applied stress, followed by gliadins and glutenins. 


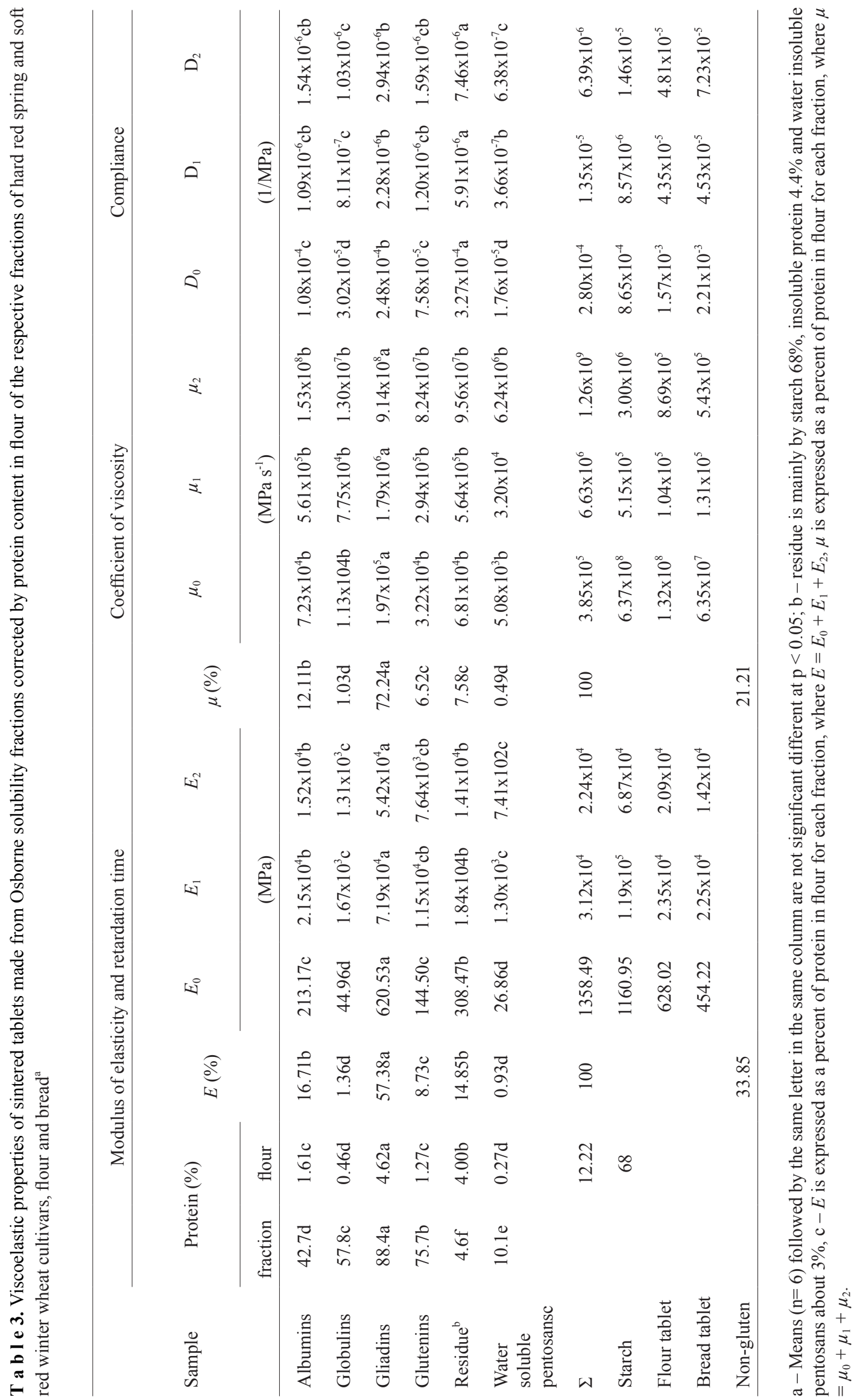




\section{ACKNOWLEDGEMENTS}

Escalante-Aburto wishes to acknowledge the financial support received from CONACyT for her Posdoc studies at CINVESTAV with the group of Bio-organic Materials, and to Mr. Agustin Galindo from CINVESTAV for his technical assistance. Ponce-García thanks CONACyT for his postdoctoral scholarship.

Conflict of interest: The Authors declare they have no conflict of interest.

\section{REFERENCES}

AACC International, 2011. Method 10-09.01. Basic straightdough bread-baking method-long fermentation. AACC International, St. Paul, Minnesota, USA.

Belton P.S., 1999. On the elasticity of wheat gluten. J. Cereal Science, 29, 103-107.

Bloksma A.H. and Bushuk, W., 1988. Rheology and chemistry of dough. In: Wheat Chemistry and Technology II (Ed Y. Pomeranz). AACCI, St. Paul, Minnesota, USA.

Chen C.H. and Bushuk W., 1970. Nature of proteins in triticale and its parental species I. Solubility characteristics and amino acid composition of endosperm proteins. Canadian J. Plant Sci., 50, 9-14.

D’Appolonia B.L. and Gilles K.A., 1971. Pentosans associated with gluten. Cereal Chemistry, 48, 428-436.

Delwiche S.R., 1998. Protein content of single kernels of wheat by Near-Infrared Reflectance Spectroscopy. J. Cereal Sci., 27, 241-251.

Dobraszczyk B.J. and Morgenstern M.P., 2003. Rheology and the breadmaking process. J. Cereal Sci., 38, 229-245.

Figueroa J.D.C, Escalante-Aburto A., Véles-Medina J.J., Hernández-Estrada Z.J., Rayas-Duarte P., Simsek S., and Ponce-García N., 2016. Viscoelastic properties of tablets from Osborne solubility fraction, pentosans and flour and bread using relaxation tests. J. Cereal Science, 69, 207-212.

Figueroa J.D.C., Hernández Z.J.E., Véles M.J.J., Rayas-Duarte P., Martínez-Flores H.E., and Ponce-García N., 2011. Evaluation of degree of elasticity and other mechanical properties of wheat kernels. Cereal Chemistry, 88, 12-18.

Figueroa J.D.C., Maucher T., Reule W., and Peña, R.J., 2009. Influence of high molecular weight glutenins on viscoelastic properties of intact wheat kernel and relation to functional properties of wheat dough. Cereal Chemistry, 86, 139-144.

Gianibelli M.C., Larroque O.R., McRitchie F., and Wrigley C.W., 2001. Biochemical, genetic, and molecular characterization of wheat glutenin and its components subunits. Cereal Chemistry, 78, 635-646.

Greenwood G.T. and Ewart J.A.D., 1975. Hypothesis for the structure of glutenin in relation to rheological properties of gluten and dough. Cereal Chemistry, Supplement 3, 146r-153r.

Haddad Y., Benet J.C., Delenne J.Y., Mermet A., and Abeccassis J., 2001. Rheological behavior of wheat endosperm - Proposal for classification based on the rheological characteristics of endosperm test samples. J. Cereal Sci., 34, 105-131.

Hernández-Estrada Z.J., Figueroa J.D.C., Rayas-Duarte P., and Morales-Sánchez E., 2014. Creep recovery tests to measure the effects of wheat glutenins on doughs and the relationships to rheological and breadmaking properties. J. Food Eng., 143, 62-68.

Hernández-Estrada Z.J., Figueroa J.D.C., Rayas-Duarte P., and Peña R.J., 2012. Viscoelastic characterization of glutenins in wheat kernels measured by creep test. J. Food Engineering, 113, 19-26.

Hernández-Estrada, Z.J. Rayas-Duarte, P. Figueroa, J.D.C., 2017. Creep recovery of wet gluten and high-molecularweight glutenin subunits composition: Relationship with viscoelasticity of dough and breadmaking quality of hard red winter wheat. Cereal Chemistry, 94, 223-229.

Jelaca J.L. and Hlynka, I., 1971. Water binding capacity of wheat flour crude pentosans and their relation to mixing characteristics of dough. Cereal Chemistry, 48, 211-222.

Khatkar B.S. and Schofield J.D., 1997. Molecular and physicochemical basis of breadmaking-properties of wheat glutenin proteins: A critical appraisal. J. Food Sci. Technol., 34, 85-102.

Li W., Dobraszczyk B.J., Dias A., and Gil A.M., 2006. Polymer conformation structure of wheat proteins and gluten subfractions revealed by ATR-FTIR. Cereal Chemistry, 83, 407-410

Lindsay M.P. and Skerritt J.H., 1999. The glutenin macropolymer of wheat flour doughs: Structure-function perspectives. Trends Food Sci. Technol., 10, 247-253.

Maucher T., Figueroa J.D.C., Reule W., and Peña J., 2009. Influence of low molecular weight on viscoelastic properties of intact wheat kernels and their relation to functional properties of wheat dough. Cereal Chemistry, 86, 372-375.

Mohsenin N.N., 1986. Physical properties of plant and animal materials. Structure, physical characteristics and mechanical properties. Gordon and Breach Science, New York, USA.

Ponce-García N., Ramírez-Wong B., Torres-Chávez P., Figueroa-Cárdenas J.D., Serna-Saldivar S.O., CortezRocha M.O., and Escalante-Aburto A., 2016. Evaluation of visco-elastic properties of conditioned wheat kernels and their doughs using a compression test under small strain. J. Sci. Food Agric., 97, 1235-1243.

Santos D.M.J., Monteiro S.R., and da Silva J.A.L., 2005. Small strain viscoelastic behaviour of wheat gluten-pentosans mixtures. European Food Res. Technol., 221, 398-405.

Saxena C.D., Rao U.J.S.P., and Rao P.H., 1997. Indian wheat cultivars: correlation between quality of gluten proteins, rheological characteristics of dough and tandoori roti quality. J. Sci. Food Agric., 74, 265-272.

Staniforth J.N. and Patel C.I., 1989. Creep compliance behavior of direct compression excipients. Powder Technol., 57, 83-87.

Žilić S., Barać M., Pešić M., Dodig D., and Ignjatović-Micić D., 2011. Characterization of proteins from grain of different bread and durum wheat genotypes. Int. J. Molecular Sci., 12, 5878-5894. 